\title{
Commentary: History of the ribosome and the origin of translation
}

\author{
Derek Caetano-Anollés ${ }^{1}$ and Gustavo Caetano-Anollés ${ }^{2 *}$ \\ ${ }^{1}$ Department of Evolutionary Genetics, Max-Planck-Institut für Evolutionsbiologie, Plön, Germany, ${ }^{2}$ Evolutionary \\ Bioinformatics Laboratory, Department of Crop Sciences, University of Illinois, Urbana, IL, USA
}

Keywords: molecular evolution, rRNA, insertion, molecular growth, cladistics, ad hocness, homoplasy, accretion

A commentary on

History of the ribosome and the origin of translation

by Petrov, A. S., Gulen, B., Norris, A. M., Kovacs, N. A., Bernier, C. R., Lanier, K. A., et al. (2015). Proc. Natl. Acad. Sci. U.S.A. 112, 15396-115401. doi: 10.1073/pnas.1509761112

Since Hennig (1966), cladistics offers a solid framework for conducting historical explorations in biology. Cladistic methodology has been successfully applied to the study of molecular evolution. For example, phylogenetic characters describing the length and stability of RNA helical segments or the abundance of protein structural domains in genomes have been used to build phylogenetic trees of molecules and proteomes, respectively (reviewed in Caetano-Anollés and Caetano-Anollés, 2015a). These methodologies have been recently extended to study the origin and evolution of the ribosome (Harish and Caetano-Anollés, 2012). In general, selecting useful molecular characters requires an assumption that they represent homologies, relationships that can be falsified within the congruent character set. This demands selecting an optimal phylogenetic tree by minimization of ad hoc hypotheses of multiple origins (homoplasy) in the ensemble of all possible unrooted trees

\section{OPEN ACCESS} and choosing an appropriate transformation series or model to unfold transformational change in them. Trees are then rooted $a$ posteriori by identifying the ancestral and derived transformational homologs. These multiple interrelationships make the entire retrodiction enterprise a challenging endeavor of reciprocal fulfillment.

Despite the usefulness of phylogenetic reconstruction and over 170 years of conceptual advances following Richard Owen's structural interpretation of homology, many continue to make evolutionary inferences with definitions of homology that are independent of history. Recently, a group of supporters of the ancient "RNA world" theory devised an algorithm that subjectively guarantees the structural origin of the ribosome in its biosynthetic RNA heart, the peptidyl transferase center (PTC) (Petrov et al., 2014). Their method assumes the universal ribosomal core evolved by gradual insertion of "branch" helices onto preexisting, coaxially-stacked, "trunk" helices, growing the rRNA molecules outwards from the PTC and leaving behind "insertion fingerprint" (IF) constrictions in their junctions. Figure 1 describes how trees of living "molecular fossils" generated by the outward growth algorithm mimic phylogenetic trees. In these trees, the nodes represent extant rRNA junctions, not hypothetical ancestral entities. The branches are not evolving taxa. Instead they represent connecting "trunk" helical segments. For the structural tree to resemble a phylogenetic tree that complies with the outward growth algorithm, "trunk" and "branch" helices must represent alternative states of a phylogenetic character uniquely describing each ribosomal junction. Similarly, the "trunk" state must always be ancestral and must change to the derived "branch" state in at least one of the branches arising from each split of the tree (Figure 1A). Note that the algorithm forbids both sister branches preserving the ancestral state, an assumption that is unrealistic in phylogenetic analysis. To simplify the tree descriptions of complex rRNA molecules, we do not show branches with derived states unless a "branch" helix turns into a "trunk" helix in a more outward region of the molecule (Figure 1B).

In previous correspondence, one of us challenged both the veracity of branch-to-trunk growth and the historical significance of IFs, arguing that they likely arise from biophysical constraints of the molecules (Caetano-Anollés, 2015). While our objections remain basically unanswered (see 


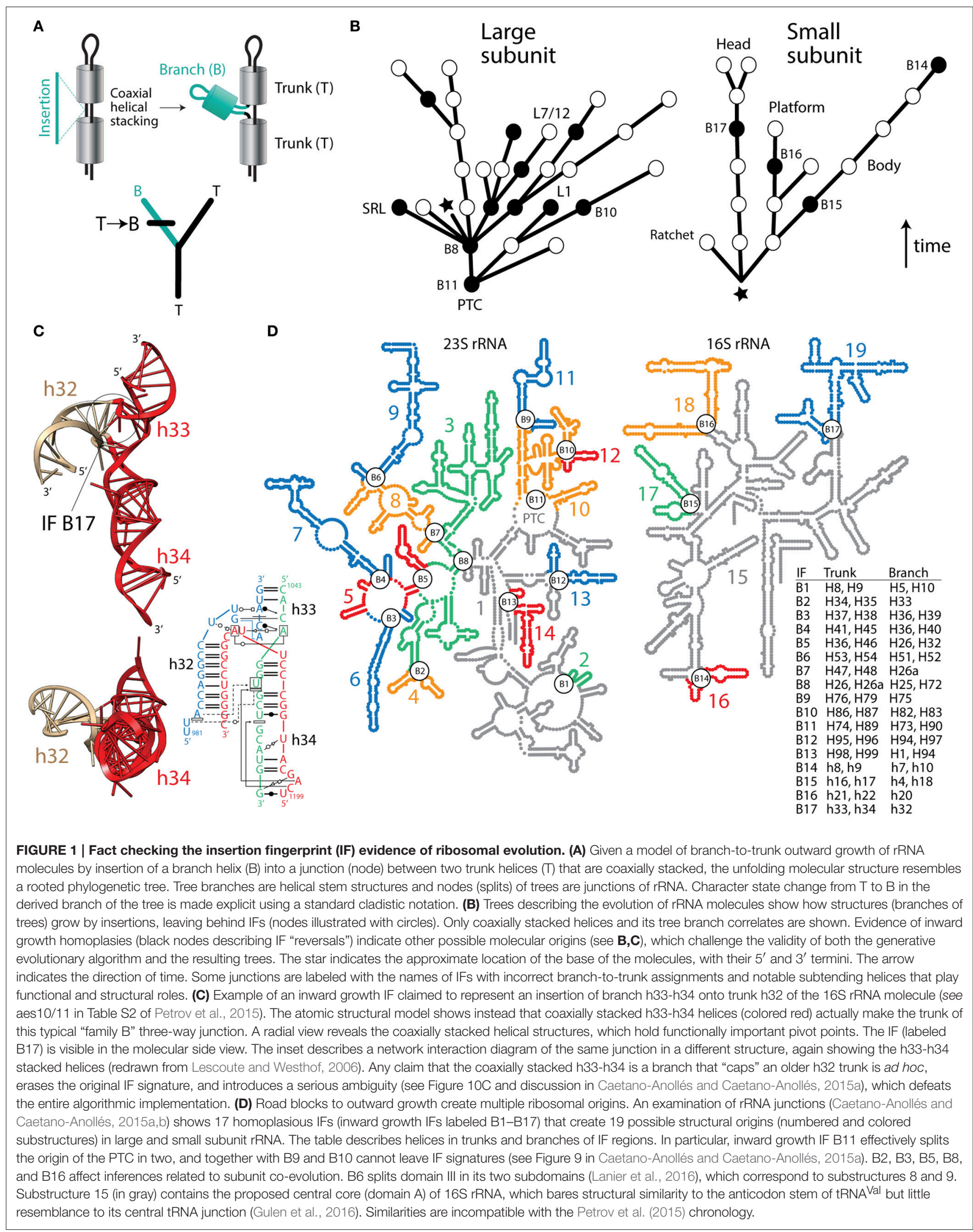


Caetano-Anollés and Caetano-Anollés, 2015b), in a recent follow up paper, Petrov et al. (2015) use the same algorithm to extend their evolutionary inferences to the small ribosomal subunit. Here, we highlight the perils of systematically disposing of evidence with formal and argumentative ad hoc hypotheses to salvage pre-falsified theory.

As stated by Farris (1984), "Science requires that choice among theories be decided by evidence, and the effect of an ad hoc hypothesis is precisely to dispose of an observation that otherwise would provide evidence against a theory. If such disposals were allowed freely, there could be no effective connection between theory and observation, and the concept of evidence would be meaningless" (Farris, 1984). Here, Farris refers to the need of minimizing ad hoc hypotheses of homoplasy when reconstructing history. Over decades, this rationale developed into modern phylogenetic analyses. Current computational methods search the space of competing historical hypotheses with optimality criteria, attempting to overthrow both hypotheses of history and homology using the hypotheticodeductive method.

In contrast, the algorithm of Petrov et al. (2014, 2015) is inductive-it demands a single molecular origin and absence of roadblocks to outward growth (homoplasies) that would create new origins, including graft-assembly from pieces and inward growth by helix reformation (discussed in Caetano-Anollés and Caetano-Anollés, 2015b). Every possible roadblock requires an additional ad hoc hypothesis to explain it, which together with dubious auxiliary assumptions (onion ribosomal growth, unbudgeted helix growth, and many other "external indicators of relative age"), weaken their theory of ribosomal history. Recently, we examined putative IFs in small and large rRNA subunits (Caetano-Anollés, 2015; Caetano-Anollés and Caetano-Anollés, 2015a,b). We showed concerning "reversals," incorrect branchto trunk assignments, none of which Petrov et al. $(2014,2015)$ explain. Figure 1C illustrates one example of these roadblocks with a coaxially stacked "trunk" listed as "branch" in Table S2

\section{REFERENCES}

Caetano-Anollés, D., and Caetano-Anollés, G. (2015b). Ribosomal accretion, apriorism and the phylogenetic method: a response to Petrov and Williams. Front. Genet. 6:194. doi: 10.3389/fgene.2015.00194

Caetano-Anollés, G. (2015). Ancestral insertions and expansions of rRNA do not support an origin of the ribosome in its peptidyl transferase center. J. Mol. Evol. 80, 162-165. doi: 10.1007/s00239-015-9677-9

Caetano-Anollés, G., and Caetano-Anollés, D. (2015a). Computing the origin and evolution of the ribosome from its structure - uncovering processes of macromolecular accretion benefiting synthetic biology. Comput. Struct. Biotechnol. J. 13, 427-447. doi: 10.1016/j.csbj.2015.07.003

Farris, J. S. (1984). "The logical basis of phylogenetic analysis," in Advances in Cladistics, Vol. 2, Proceedings of the Second Meeting of the Willi Hennig Society, eds N. I. Platnick and V. A. Funk (New York, NY: Columbia University Press), $7-36$.

Gulen, B., Petrov, A. S., Okafor, C. D., Vander Wood, D., O’Neill, E. B., Hud, N. V., et al. (2016). Ribosomal small subunit domains radiate from a central core. Sci. Rep. 6:20885. doi: 10.1038/srep20885

Harish, A., and Caetano-Anollés, G. (2012). Ribosomal history reveals origins of modern protein synthesis. PLoS ONE 7:e32776. doi: 10.1371/ journal.pone.0032776

Hennig, W. (1966). Phylogenetic Systematics. Urbana, IL: University of Illinois Press. of Petrov et al. (2015). Ad hoc dismissals of this kind include at least 17 branch-to-trunk homoplasies (Caetano-Anollés and Caetano-Anollés, 2015b), which create 19 possible origins for rRNA molecules (Figure 1D), including the split of the PTC at its core (Caetano-Anollés and Caetano-Anollés, 2015a,b). Two of these possible origins, the core and tail subdomains of Domain III of 23S rRNA (segments 8 and 9, Figure 1D), fold autonomously, together or in isolation (Lanier et al., 2016). Thus, structure and biophysics are in line with homoplasy-based evidence and not the outward growth model.

To summarize, reconstructing ribosomal history from IF evidence is impossible in absence of: (i) trees describing RNA structural evolution, (ii) a model of evolutionary change for optimization of those changes on the trees, and (iii) a processfree rooting criterion. More importantly, the algorithm cannot confirm nor deny the historical validity of IF evidence, since IFs are not homologies testable on trees. Thus, the work of Petrov et al. $(2014,2015)$ illustrates the perils of ad hocness in the study of ribosomal evolution. Assumptions should never be used to salvage theory and canonize false facts. If so, the search for truth in science would rapidly morph into narratives of persuasion and mythology. More troubling however is the use of an algorithmic implementation of homology that is history-independent despite half a century of cladistic developments.

\section{AUTHOR CONTRIBUTIONS}

All authors listed, have made substantial, direct and intellectual contribution to the work, and approved it for publication.

\section{FUNDING}

Computational biology in the Evolutionary Bioinformatics laboratory is supported by grants from NSF (OISE-1172791) and USDA (ILLU-802-909). DC is recipient of NSF postdoctoral fellowship award 1523549.

Lanier, K. A., Athavale, S. S., Petrov, A. S., Wartell, R., and Williams, L. D. (2016). Imprint of ancient evolution on rRNA folding. Biochemistry 55, 4603-4613. doi: 10.1021/acs.biochem.6b00168

Lescoute, A., and Westhof, E. (2006). Topology of three-way junctions in folded RNAs. RNA 12, 83-93. doi: 10.1261/rna.2208106

Petrov, A. S., Bernier, C. R., Hsiao, C., Norris, A. M., Kovacs, N. A., Waterbury, C. C., et al. (2014). Evolution of the ribosome at atomic resolution. Proc. Natl. Acad. Sci. U.S.A. 111, 10251-10256. doi: 10.1073/pnas.1407205111

Petrov, A. S., Gulen, B., Norris, A. M., Kovacs, N. A., Bernier, C. R., Lanier, K. A., et al. (2015). History of the ribosome and the origin of translation. Proc. Natl. Acad. Sci. U.S.A. 112, 15396-115401. doi: 10.1073/pnas.15097 61112

Conflict of Interest Statement: The authors declare that the research was conducted in the absence of any commercial or financial relationships that could be construed as a potential conflict of interest.

Copyright (c) 2017 Caetano-Anollés and Caetano-Anollés. This is an open-access article distributed under the terms of the Creative Commons Attribution License (CC BY). The use, distribution or reproduction in other forums is permitted, provided the original author(s) or licensor are credited and that the original publication in this journal is cited, in accordance with accepted academic practice. No use, distribution or reproduction is permitted which does not comply with these terms. 